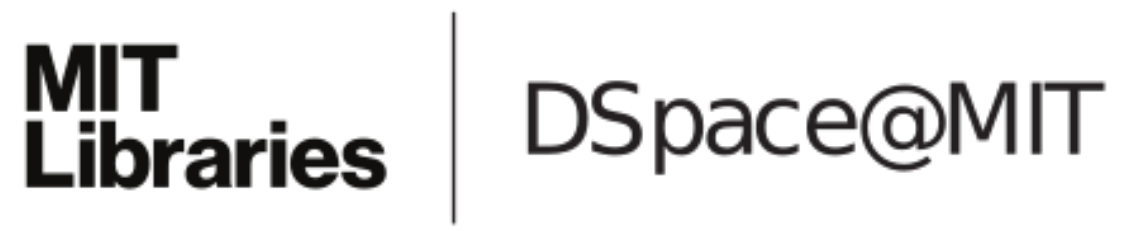

\author{
MIT Open Access Articles
}

\section{Mechanisms of cooperation in cancer nanomedicine: towards systems nanotechnology}

The MIT Faculty has made this article openly available. Please share how this access benefits you. Your story matters.

Citation: Hauert, Sabine, and Sangeeta N. Bhatia. "Mechanisms of Cooperation in Cancer Nanomedicine: Towards Systems Nanotechnology." Trends in Biotechnology 32, no. 9 (September 2014): 448-455.

As Published: http://dx.doi.org/10.1016/j.tibtech.2014.06.010

Publisher: Elsevier

Persistent URL: http://hdl.handle.net/1721.1/99871

Version: Author's final manuscript: final author's manuscript post peer review, without publisher's formatting or copy editing

Terms of use: Creative Commons Attribution 
Published as: Trends Biotechnol. 2014 September ; 32(9): 448-455.

\title{
Mechanisms of Cooperation in Cancer Nanomedicine: Towards Systems Nanotechnology
}

\author{
Sabine Hauert ${ }^{a, b, c}$ and Sangeeta N. Bhatia $a^{a, b, d, e, f}$ \\ aHarvard-MIT Division of Health Sciences and Technology, Institute for Medical Engineering and \\ Science, Massachusetts Institute of Technology, Cambridge, MA 02139, USA \\ bDavid H. Koch Institute for Integrative Cancer Research, Massachusetts Institute of Technology, \\ Cambridge, MA 02139, USA \\ cEngineering Mathematics, University of Bristol, Bristol BS8 1TR, UK \\ dElectrical Engineering and Computer Science, Massachusetts Institute of Technology, \\ Cambridge, MA 02139, USA \\ eDepartment of Medicine, Brigham and Women's Hospital, Boston, MA 02115, USA \\ fHoward Hughes Medical Institute, Massachusetts Institute of Technology, Cambridge, MA \\ 02139, USA
}

\begin{abstract}
Nanoparticles are designed to selectively deliver therapeutics and diagnostics to tumors. Their size, shape, charge, material, coating and cargo, determine their individual functionalities. A systems approach could help predict the behavior of trillions of nanoparticles interacting in complex tumor environments. Engineering these nanosystems may lead to biomimetic strategies where interactions between nanoparticles and their environment give rise to cooperative behaviors typically seen in natural self-organized systems. Examples include nanoparticles that communicate the location of a tumor to amplify tumor homing, or self-assemble and disassemble to optimize nanoparticle transport. The challenge is to discover which nanoparticle designs lead to a desired systems behavior. To this end, novel nanomaterials, deep biological understanding, and computational tools are emerging as the next frontier.
\end{abstract}

\section{Keywords}

Swarming; Cooperation; Nanoparticles; Systems Nanotechnology; Cancer

(c) 2014 Elsevier Ltd. All rights reserved.

Corresponding author: Bhatia, S.N. (sbhatia@mit.edu).

Publisher's Disclaimer: This is a PDF file of an unedited manuscript that has been accepted for publication. As a service to our customers we are providing this early version of the manuscript. The manuscript will undergo copyediting, typesetting, and review of the resulting proof before it is published in its final citable form. Please note that during the production process errors may be discovered which could affect the content, and all legal disclaimers that apply to the journal pertain. 


\section{Cooperative nanosystems}

Cancer is one of the leading causes of death worldwide. To address cancer, bioengineers are designing nanoparticles that can deliver treatments and diagnostics selectively to tumors [1, 2]. Their size, typically between $5 \mathrm{~nm}$ and $500 \mathrm{~nm}$, allows them to escape the leaky vessels in tumors [3]. Focus has been on engineering the functionalities of individual nanoparticles to: improve transport [4], target the tumor vasculature [5, 6] or extracellular matrix [7], deliver therapeutics $[8,9]$, diagnostics $[10]$ or heat $[11,12]$ to the tumor environment, and reprogram cancer cells [13] or the immune system [14].

The behavior of each nanoparticle depends on its design and the resulting interactions in the body. The collective behavior of trillions of such nanoparticles interacting in a complex tumor environment can define their success as diagnostic or treatment agents [15]. Predicting and engineering these collective behaviors is empirical and not intuitive. For example, nanoparticles that are optimized to strongly bind and accumulate in cancer cells mostly accumulate in the first cells they encounter after leaking into the tumor environment. The resulting collective behavior is poor tissue penetration, with deep seeded tumor cells left untreated, otherwise called a binding site barrier [16-18]. Weaker nanoparticle binding, although detrimental to the function of the individual nanoparticle, could lead to a better system outcome. Further engineering these behaviors could result in emergent cooperative behaviors typically seen in self-organized systems.

Self-organized systems in nature, including social insects, animals, humans and cells, are able to perform complex behaviors, such as amplification, optimization, mapping, structure assembly, collective motion, synchronization and decision making, through the local interactions of many simple agents and their environment [19-22]. The field of swarm robotics $[23,24]$ has long taken inspiration from nature to engineer minimal robots that use simple rules to interact with their neighbors and local environment to solve complex real world problems [25-27]. Similarly, behaviors demonstrated in nanomedicine include: selfassembly of nanoparticles to anchor imaging agents in tumors [28, 29], disassembly of nanoparticles to increase tissue penetration [30,31], nanoparticles that compute the state of a tumor [32], nanoparticle-based remodeling of tumor environments to improve secondary nanoparticle transport [33], or nanoparticle signaling of tumor location to amplify the accumulation of other nanoparticles in tumors [34].

Even for simple nanoparticle designs, engineering and predicting the collective behavior of large numbers of nanoparticles that interact with complex tumor environments is nonintuitive. Through a systems approach, bioengineers could automatically explore nanoparticle designs using crowdsourcing (http://nanodoc.org) and machine learning [35]. Bioengineers could: model the resulting collective behavior in simulation $[4,16,17,36]$, automatically test the best candidates experimentally through fast prototyping of both the nanoparticles [37, 38] and their environment [39], and finally validate the collective behaviors in vivo with feedback provided by high-resolution imaging [40]. Expertise in nanomaterials, deep understanding of cancer biology, and advances in the modeling and automation of nanosystems are making possible the first step in this direction. 
Lessons learned from the design of cooperative nanosystems could also prove useful in the engineering of natural swarmers, such as cells of the immune system [41] or synthetic bacteria [42], to improve tumor treatment and diagnostics. Overall, a systems approach to understand and engineer self-organized systems has the potential to result in behaviors that go beyond the functionalities of the individual agents and towards efficient, modular and predictable outcomes.

\section{Nanoparticle behaviors}

A large variety of nanoparticles have been engineered to deliver therapies and diagnostics to tumors. The design of a nanoparticle, and its interactions with the environment, defines its behavior. The composition of each nanoparticle can be summarized by its main features in terms of initial size, shape, charge, coating, cargo, and material, the combination of which determine its ability to move and interact with the environment (Figure 1) [43, 44].

The size of a nanoparticle has been shown to influence its circulation time, extravasation, interstitial diffusion and ability to be internalized by cells [45, 46]. Nanoparticles smaller than $5 \mathrm{~nm}$ tend to escape the blood stream into tissues throughout the body $[47,48]$. They are rapidly filtered by the kidneys and cleared in the urine. As a result, small nanoparticles have a short circulation time (on the order of minutes), rapid entry into tumors, and fast diffusion through the tumor tissue. Nanoparticles that reach the edge of a solid tumor can be carried out due to the gradient in hydrostatic pressure caused by the tumor physiology relative to the surrounding healthy tissue, thereby making their retention more challenging [3]. Nanoparticles larger than $5 \mathrm{~nm}$ and up to $500 \mathrm{~nm}$ are, in some cases, able to remain in circulation for a longer time and accumulate in solid tumors by escaping through the enlarged pores in angiogenic vessels. Such passive targeting of nanoparticles due to porous angiogenic endothelium and dysfunctional lymphatic drainage, observed in some tumors, has been called the enhanced permeability and retention (EPR) effect [49]. Once in the tumor, the slow diffusion of larger nanoparticles and the difficulty of navigating the extracellular matrix tend to limit their ability to penetrate tumor tissue [50]. The size of a nanoparticle also limits its ability to be internalized by cells, and data indicates that different sizes could be trafficked via different endocytic pathways [44].

The shape and modulus of nanoparticles can also impact cellular uptake [51]. Nanoparticles with high aspect ratios, and particles that are rigid, have been shown to accumulate more slowly in macrophages than smaller, flexible particles, thereby reducing their clearance time from the blood. In situations where uptake is important, such as in tumor environments, spherical nanoparticles have thus far proven more efficient.

The charge of a nanoparticle also impacts its circulation time. Charged nanoparticles are rapidly opsonized and cleared by the immune system [52]. Passivating coatings such as polyethylene glycol have been used to shield the charge of nanoparticles, improve their circulation time on the order of days, and result in their accumulation in tumor tissue [43]. Similarly, phagocytic uptake can be minimized by coating nanoparticles with a "selfpeptide" derived from the human "don't eat me" receptor, CD47 [53]. Once in tumor tissue, charged nanoparticles are preferentially taken up by tumor cells relative to uncharged 
particles. Researchers have been addressing this dichotomy by designing nanoparticles that shed their neutral coatings to display a charged interior upon entering the tumor environment based on pH or enzymatic activity [54-56].

Beyond charge, nanoparticle surfaces can be engineered to display targeting ligands [5, 7, 57] including peptides [58], antibodies [59], aptamers [60] and small molecules [61]. Receptors that are expressed at high levels on certain tumor cells can serve as targets that drive the binding and intracellular delivery of nanoparticles [6]. For small nanoparticles, targeting has proven useful in anchoring nanoparticles in the tumor environment, resulting in increased accumulation over time [62]. Recently, nanoparticles decorated with ligands expressing a CendR domain have been shown to activate the neuropilin-1 receptor and subsequently increase tissue penetration by initiating a trancellular, active transport pathway [63]. The effect can be made specific to tumor tissue by adding a targeting domain to the peptide. Receptors overexpressed on angiogenic endothelial cells have been used as vascular 'zip codes' to direct and capture circulating nanoparticles and concentrate them in tumor environments [6], or can serve as an antiangiogenic target towards the normalization of the vascular bed [3,64]. Nanoparticles have also been engineered to target the extracellular matrix (ECM), either to improve the retention of nanoparticles [65] or degrade the ECM to improve drug permeation in tumors [66]. Finally, nanoparticles can be engineered to target $\mathrm{T}$ cells and enhance immune responses towards tumors [14, 67].

One other design benefit that nanoparticles can offer is the capacity to shield materials that would otherwise be toxic or degraded in the body. The therapeutic cargo may then be carried to the tumor where it will be released or activated prior to local application, thereby minimizing systemic side effects while improving effectiveness despite low dosages. Cargos transported by nanoparticles include: chemotherapies [8,9] and siRNA for knockdown of gene expression in tumor cells [13, 68], antiangiogenic agents [64, 69], agents that are disruptive to the ECM [70], imaging agents [1, 10], or adjuvants to activate the immune system [14].

The ability of nanoparticles to react to their environment is directly connected to their material composition [71]. Nanoparticles can be engineered to release cargos in an openloop manner at a predetermined rate based on material erosion, or cargo diffusion through the nanoparticle matrix or pores [72-74]. To increase the level of control, materials can release their cargo or change their physical properties in response to local stimuli in tumor microenvironments, such as $\mathrm{pH}$ or enzymatic activity [55, 75-77]. Energy sensitive materials can be activated by global signals such as magnetic fields [78-80], sound [81] or light $[11,82,83]$, which can also power them to move, emit light or heat, or to release a cargo.

Overall, the ability of nanoparticles to sense, move and act in the body is determined by their design and interactions with the environment. Engineering the behavior of nanoparticles has become increasingly possible, thanks to a deepening understanding of biology and nanoparticle transport, and the impressive toolbox of nanoparticle modifiers available to bioengineers. 


\section{Collective behaviors}

In other fields, engineering collective behavior is necessary to achieve a desired system outcome. Engineering the collective behavior of nanoparticles may similarly lead to a better system outcome. Most nanoparticle systems implicitly cooperate, and each nanoparticle is designed to optimize its individual functionality [84]. The collective impact of the nanoparticles as treatment or imaging agents is assumed to be the sum of the independent nanoparticle effects. Understanding the system-level behavior of implicit cooperators may add insight that improves predictions. Emphasis could be placed on studying whether the nanoparticles can collectively distribute throughout a tumor environment or accumulate at effective levels in, or around, targeted cells [4]. Similarly, combination therapies aimed at preventing resistance can be composed of different types of nanoparticles that independently target cells in the tumor $[85,86]$.

Nanoparticles that physically interact have a more direct means of cooperation. These systems typically self-assemble or disassemble to modify their kinetics or collectively transport combined treatment and imaging agents to tumors. Rapidly diffusing imaging agents are able to anchor in tumors by binding to previously injected gold nanoparticles that have had time to accumulate there due to the EPR effect [29]. Similarly, small $(10 \mathrm{~nm})$ gold nanoparticles engineered to release conjugated doxorubicin in acidic tumor environments, can subsequently self-assemble to form larger gold aggregates that are then used for photothermal therapy $[28,87]$. In vitro experiments show how nanoparticles that selfassemble in response to enzymatic activity may be able to perform logic computations towards the diagnosis of tumor state [88]. Larger nanoparticles $(100 \mathrm{~nm})$ are able to disassemble into smaller nanoparticles once inside the tumor environment, in response to enzymatic activity, thereby improving their circulation time, accumulation in the tumor, and ability to penetrate deep in the tissue [31]. Other multi-stage nanoparticles including nested nanoparticles, mother ships, and nanocells are able to overcome transport barriers through the release of nano-based components in tumor environments [64, 89, 90].

In contrast to collective behaviors mediated by direct interactions between nanoparticles, many swarm systems found in nature communicate by modifying the environment. This concept is called stigmergy [19]. Ants deposit and sense chemical signals to form trails to sources of food [20]. Termites are able to build complex structures by modifying and locally sensing their physical environment [27]. In a similar way, nanoparticles have been designed to modify their physical environment or deposit chemical signals. Gold nanorods that accumulate in a tumor, upon heating with NIR light to sub-lethal temperatures, can improve perfusion of angiogenic vessels and in some cases upregulate receptors used in targeting, which in turn improves the delivery of a second wave of nanoparticles, such as liposomes and magnetic nanoworms, to tumors for treatment and imaging purposes [33, 82]. Similarly, nanoparticles that aim to normalize the vascular bed, or degrade the extracellular matrix can improve the transport of secondary nanoparticles $[3,66]$.

Nanoparticles can also communicate through the environment by depositing a signal according to two possible modes of action. In the first, nanoparticles are able to release either a cargo or energy, which can directly interact with other nanoparticles. This approach 
offers an important advantage: nanoparticle communication can be engineered to be orthogonal to the host system. As an example, gold nanorods activated through NIR light emit heat in tumors to trigger the release of chemotherapeutics contained in thermally sensitive drug carriers [91]. Unfortunately, the scales at which nanoparticles function make it difficult for a chemical cargo to encounter a second nanoparticle in the environment. In contrast, biological systems have built-in mechanisms to amplify signals, coercing biological cascades can result in longer-range communication systems. Gold nanorods heated through NIR light can cause a clotting cascade in tumors [34]. This biological cascade serves as a signal to communicate the location of the tumor to circulating nanoparticles loaded with chemotherapeutics. To achieve this outcome, the receiving nanoparticle, here a liposome or nanoworm, is targeted to a byproduct of the coagulation cascade (the transglutaminase FXIII). This communication strategy leads to a 40 -fold increase in the amount of chemotherapeutic delivered to the tumor when compared to a noncommunicating system [34].

Nanoparticles can cooperate implicitly, directly through self-assembly and disassembly, or through stigmergy (Figure 2). These behaviors have been useful to improve nanoparticle transport, accumulation, and distribution in tumor tissues towards treatment and diagnostic applications.

\section{Systems nanotechnology}

A systems approach could help engineer collective behaviors for nanoparticles (Figure 3) [92]. Currently, most nanoparticle systems are engineered based on the desired functionalities of the individual nanoparticles. Designing cooperative behaviors requires bioengineers to initially define the behavior that should be displayed by the nanoparticle collective within their environment of action. Examples include: "accumulate in all tumor cells at lethal levels, assuming sites of extravasation are no further than 200um from the cells" [16]. The challenge is then to understand which individual nanoparticle designs could give rise to this collective behavior.

Currently, the exploration of nanoparticle designs is conducted by experts with a deep knowledge of the intricacies of cancer nanomedicine. This paradigm is ideal when the collective behavior resulting from a given nanoparticle design and its iterations are well understood. When new designs are needed, or more complex collective behaviors are envisaged, automatic exploration tools built around machine learning and crowdsourcing can help. Mathematical optimization was used to explore formulations, in terms of binding kinetics and diffusion, which would enable targeted nanoparticles to penetrate deep into tumor tissues [16]. Regression analysis was used to explore the impact of nanoparticle structure on their ability to deliver siRNAs to tumor cells [93]. When the search space is too large, the power of the crowd can enable human guided exploration of nanoparticle designs. Crowdsourcing has been shown in the past to find solutions to complex scientific problems that were intractable for computers, despite their impressive processing power [94]. NanoDoc (http://nanodoc.org), for example, provides an online tool to crowdsource the design of nanoparticles. Using an iterative approach, players design nanoparticle treatments, 
inject them into a virtual tumor, and continuously improve their design until they are able to achieve a desired collective behavior.

Once provided with a nanoparticle design, computer simulations and mathematical models can help predict their collective behavior. Analytical and Monte Carlo simulations have been used to predict the impact of multivalency of targeting moieties on the super-selectivity of nanoparticles to cancer cells [95]. Modeling the kinetics of nanoparticle populations in tumors has been demonstrated by several research groups using stochastic and deterministic approaches [16-18, 36, 96]. The models describe how nanoparticles extravasate, diffuse through the interstitial space, and bind to, or are internalized by cancer cells. Ideally, iterations between nanoparticle design and the resulting simulated behavior give rise to general guidelines that can be applied to nanosystems without the need to iterate at length experimentally.

After developing plans for an idealized nanoparticle, these designs must then be engineered. Most often, this step requires deep expertise, based on the state-of-the-art in the field. However, automatic tools can help combine nanoparticle features, such as size, coating and cargo in a deterministic fashion [37]. Particle replication in non-wetting templates (PRINT) technology offers an easy to use platform for the design and fabrication of monodisperse particles from a wide range of matrices with complete control over the physicochemical properties of the particle [38]. Combinatorial approaches in designing and synthesizing polymeric systems enable high-throughput synthesis and screening of nanoparticles with desired drug loading, retention in circulation and targeting. For this purpose, a number of polymer libraries have been designed [97-99]. Recent work on DNA self-assembly also enables the rapid prototyping of nanoscale structures [100-103].

Testing the collective behavior of nanoparticles is made challenging by the fact that the environment plays such an important role in the rise of emergent behaviors. In vivo experiments are an ideal testbed, provided that high-resolution imaging can be performed during the course of the experiment. Multimodal studies of tumor pathophysiology and nanoparticle dynamics can be performed with the help of intravital microscopy in window chambers $[104,105]$. When in vivo experiments are not feasible, micro-devices, capable of capturing essential features of the tumor environment that are necessary for the emergence of collective nanoparticle behaviors can find utility. A tumor-on-a-chip system, in which a tumor-like spheroid is placed in a microfluidic channel, enables real-time analysis of nanoparticle kinetics at physiological flow conditions [106]. Recent technologies, including 3D printing, scaffolding, and cell patterning, layering and self-assembly, have enabled construction of complex 3D-human tissues, including blood and lymph capillary networks, and could be used as a testbed for cooperative nanosystems in vitro [39, 107, 108].

A key grand challenge is the integration of all the technologies needed to provide a full pipeline - from problem definition to clinical translation.

\section{Concluding remarks and future perspectives}

Nanoparticle behaviors result from their individual designs and interactions with the environment. The control of a nanoparticle is enabled by changing its shape, charge, 
material, coating and cargo. The behavior of trillions of nanoparticles in vivo determines their success as treatment and diagnostic agents. The field could therefore benefit from an increased understanding and systematic approaches to engineering the collective behavior of nanoparticles. Examples of self-organization in nature show that emergent behaviors arise when simple agents interact directly, or through the environment, and can lead to modular, scalable, and efficient strategies that outperform the capabilities of individual agents. Such concepts are already being used in other fields of engineering, such as swarm robotics.

Designing individual nanoparticles towards a desired cooperative strategy is challenging and could benefit from a systems approach. Such an endeavor would build on advances in computational exploration, simulation, high-throughput design of nanoparticles and the engineering of experimental testbeds in vitro and in vivo. This approach is now made possible thanks to advances in nanoparticle design and a deeper understanding of biology, which have given rise to the first instances of cooperative nanoparticles that can communicate tumor locations, improve transport, and self-assemble or disassemble. The next frontier is to learn from these examples to design biomedical systems that can perform complex tasks such as optimization, computation, decision-making, construction, selfassembly and collective motion. Strategies of cooperation could go beyond nanoparticles to integrate engineered biological systems including synthetic bacteria and cells of the immune system towards biomedical applications.

\section{Acknowledgments}

The authors are grateful to Dr. Fleming for her help in reviewing this manuscript. Dr. Hauert and Dr. Bhatia acknowledge support from the Human Frontier Science Program. Dr. Bhatia is an HHMI investigator.

\section{References}

1. Bao G, et al. Multifunctional nanoparticles for drug delivery and molecular imaging. Annu Rev Biomed Eng. 2013; 15:253-282. [PubMed: 23642243]

2. Davis ME, et al. Nanoparticle therapeutics: an emerging treatment modality for cancer. Nat Rev Drug Discov. 2008; 7:771-782. [PubMed: 18758474]

3. Jain RK, Stylianopoulos T. Delivering nanomedicine to solid tumors. Nat Rev Clin Oncol. 2010; 7:653-664. [PubMed: 20838415]

4. Ferrari M. Frontiers in cancer nanomedicine: directing mass transport through biological barriers. Trends Biotechnol. 2010; 28:181-188. [PubMed: 20079548]

5. Brannon-Peppas L, Blanchette JO. Nanoparticle and targeted systems for cancer therapy. Adv Drug Deliver Rev. 2012; 64:206-212.

6. Ruoslahti E, et al. Targeting of drugs and nanoparticles to tumors. J Cell Biol. 2010; 188:759-768. [PubMed: 20231381]

7. Kanapathipillai M, et al. Nanoparticle targeting of anti-cancer drugs that alter intracellular signaling or influence the tumor microenvironment. Adv Drug Deliver Rev. 2014

8. Cho K, et al. Therapeutic nanoparticles for drug delivery in cancer. Clin Cancer Res. 2008; 14:1310-1316. [PubMed: 18316549]

9. Wang AZ, et al. Nanoparticle delivery of cancer drugs. Annu Rev Med. 2012; 63:185-198. [PubMed: 21888516]

10. Ryu JH, et al. Tumor-targeting multi-functional nanoparticles for theragnosis: new paradigm for cancer therapy. Adv Drug Deliver Rev. 2012; 64:1447-1458.

11. Melancon M, et al. Cancer theranostics with near-infrared light-activatable multimodal nanoparticles. Acc Chem Res. 2011; 44:947-956. [PubMed: 21848277] 
12. Pissuwan D, et al. Therapeutic possibilities of plasmonically heated gold nanoparticles. Trends Biotechnol. 2006; 24:62-67. [PubMed: 16380179]

13. Kanasty R, et al. Delivery materials for siRNA therapeutics. Nat Mater. 2013; 12:967-977. [PubMed: 24150415]

14. Moon JJ, et al. Engineering nano- and microparticles to tune immunity. Adv Mater. 2012; 24:3724-3746. [PubMed: 22641380]

15. Taurin S, et al. Anticancer nanomedicine and tumor vascular permeability; Where is the missing link? J Control Release. 2012; 164:265-275. [PubMed: 22800576]

16. Hauert $\mathrm{S}$, et al. A computational framework for identifying design guidelines to increase the penetration of targeted nanoparticles into tumors. Nano Today. 2013; 8:566-576. [PubMed: 25009578]

17. Thurber GM, Weissleder R. A systems approach for tumor pharmacokinetics. PLoS ONE. 2011; 6:e24696. [PubMed: 21935441]

18. Wittrup KD, et al. Practical theoretic guidance for the design of tumor-targeting agents. Methods Enzymol. 2012; 503:255-268. [PubMed: 22230572]

19. Bonabeau, E., et al. Swarm intelligence: From natural to artificial systems. Oxford University Press; 1999.

20. Camazine, S., et al. Self-Organization in Biological Systems. Princeton University Press; 2003.

21. Couzin ID. Collective cognition in animal groups. Trends Cogn Sci. 2009; 13:36-43. [PubMed: 19058992]

22. Krause J, et al. Swarm intelligence in animals and humans. Trends Ecol Evol. 2010; 25:28-34. [PubMed: 19735961]

23. Sahin, E. Swarm Robotics. Springer; Berlin Heidelberg: 2005. Swarm robotics: From sources of inspiration to domains of application; p. 10-20.

24. Winfield, AFT., et al. Swarm Robotics. Springer; Berlin Heidelberg: 2005. Towards dependable swarms and a new discipline of swarm engineering; p. 126-142.

25. Hauert $S$, et al. Reynolds flocking in reality with fixed-wing robots: communication range vs. maximum turning rate. IEEE/RSJ International Conference on Intelligent Robots and Systems. 2011:5015-5020.

26. Kernbach, S. Handbook of Collective Robotics: Fundamentals and Challenges. Pan Stanford Publishing; 2013.

27. Werfel J, et al. Designing collective behavior in a termite-inspired robot construction team. Science. 2014; 343:754-758. [PubMed: 24531967]

28. Nam J, et al. pH-Induced aggregation of gold nanoparticles for photothermal cancer therapy. J Am Chem Soc. 2009; 131:13639-13645. [PubMed: 19772360]

29. Perrault SD, Chan WCW. In vivo assembly of nanoparticle components to improve targeted cancer imaging. Proc Natl Acad Sci U S A. 2010; 107:11194-11199. [PubMed: 20534561]

30. Godin B, et al. Multistage nanovectors: from concept to novel imaging contrast agents and therapeutics. Acc Chem Res. 2011; 44:979-989. [PubMed: 21902173]

31. Wong C, et al. Multistage nanoparticle delivery system for deep penetration into tumor tissue. Proc Natl Acad Sci USA. 2011; 108:2426-2431. [PubMed: 21245339]

32. Kwong GA, et al. Mass-encoded synthetic biomarkers for multiplexed urinary monitoring of disease. Nat Biotechnol. 2013; 31:63-70. [PubMed: 23242163]

33. Park JH, et al. Cooperative nanomaterial system to sensitize, target, and treat tumors. Proc Natl Acad Sci U S A. 2010; 107:981-986. [PubMed: 20080556]

34. von Maltzahn G, et al. Nanoparticles that communicate in vivo to amplify tumour targeting. Nat Mater. 2011; 10:545-552. [PubMed: 21685903]

35. Phan JH, et al. Convergence of biomarkers, bioinformatics and nanotechnology for individualized cancer treatment. Trends Biotechnol. 2009; 27:350-358. [PubMed: 19409634]

36. Florence AT. "Targeting” nanoparticles: the constraints of physical laws and physical barriers. J Control Release. 2012; 164:115-124. [PubMed: 22484196]

37. Abeylath SC, et al. Combinatorial-designed multifunctional polymeric nanosystems for tumortargeted therapeutic delivery. Acc Chem Res. 2011; 44:1009-1017. [PubMed: 21761902] 
38. Xu J, et al. Future of the particle replication in nonwetting templates (PRINT) technology. Angew Chem. 2013; 52:6580-6589. [PubMed: 23670869]

39. Vickerman V, et al. Design, Fabrication and Implementation of a Novel Multi Parameter Control Microfluidic Platform for Three-Dimensional Cell Culture and Real-Time Imaging. Lab Chip. 2008; 8:1468-1477. [PubMed: 18818801]

40. Weissleder R, Pittet MJ. Imaging in the era of molecular oncology. Nature. 2008; 452:580-589. [PubMed: 18385732]

41. Deisboeck TS, Couzin ID. Collective behavior in cancer cell populations. Bioessays. 2009; 31:190-197. [PubMed: 19204991]

42. Forbes NS. Engineering the perfect (bacterial) cancer therapy. Nat Rev Cancer. 2010; 10:785-794. [PubMed: 20944664]

43. Petros RA, DeSimone JM. Strategies in the design of nanoparticles for therapeutic applications. Nat Rev Drug Discov. 2010; 9:615-627. [PubMed: 20616808]

44. Wang J, et al. More effective nanomedicines through particle design. Small. 2011; 7:1919-1931. [PubMed: 21695781]

45. Dreaden EC, et al. Size matters: gold nanoparticles in targeted cancer drug delivery. Ther Deliv. 2012; 3:457-478. [PubMed: 22834077]

46. Kievit FM, Zhang M. Cancer nanotheranostics: improving imaging and therapy by targeted delivery across biological barriers. Adv Mater. 2011; 23:217-247.

47. Choi HS, et al. Renal clearance of quantum dots. Nat Biotechnol. 2007; 25:1165-1170. [PubMed: 17891134]

48. Longmire $\mathrm{M}$, et al. Clearance properties of nano-sized particles and molecules as imaging agents: considerations and caveats. Nanomed. 2008; 3:703-717.

49. Maeda H, et al. The EPR effect for macromolecular drug delivery to solid tumors: Improvement of tumor uptake, lowering of systemic toxicity, and distinct tumor imaging in vivo. Adv Drug Delivery Rev. 2012; 65:71-79.

50. Perrault SD, et al. Mediating tumor targeting efficiency of nanoparticles through design. Nano Lett. 2009; 9:1909-1915. [PubMed: 19344179]

51. Venkataraman S, et al. The effects of polymeric nanostructure shape on drug delivery. Adv Drug Deliver Rev. 2011; 63:1228-1246.

52. Owens DE, Peppas NA. Opsonization, biodistribution, and pharmacokinetics of polymeric nanoparticles. Int J Pharm. 2006; 307:93-102. [PubMed: 16303268]

53. Rodriguez PL, et al. Minimal "Self" peptides that inhibit phagocytic clearance and enhance delivery of nanoparticles. Science. 2013; 339:971-975. [PubMed: 23430657]

54. Harris TJ, et al. Protease-triggered unveiling of bioactive nanoparticles. Small. 2008; 4:1307-1312. [PubMed: 18690639]

55. Poon Z, et al. Layer-by-layer nanoparticles with a pH-sheddable layer for in vivo targeting of tumor hypoxia. ACS Nano. 2011; 5:4284-4292. [PubMed: 21513353]

56. Romberg B, et al. Sheddable coatings for long-circulating nanoparticles. Pharm Res. 2008; 25:5571. [PubMed: 17551809]

57. Kamaly N, et al. Targeted polymeric therapeutic nanoparticles: design, development and clinical translation. Chem Soc Rev. 2012; 41:2971-3010. [PubMed: 22388185]

58. Pearce TR, et al. Peptide targeted lipid nanoparticles for anticancer drug delivery. Adv Mater. 2012; 24:3803-3822. 3710. [PubMed: 22674563]

59. Julien DC, et al. Utilization of monoclonal antibody-targeted nanomaterials in the treatment of cancer. mAbs. 2011; 3:467-478. [PubMed: 21814040]

60. Yang L, et al. Aptamer-conjugated nanomaterials and their applications. Adv Drug Deliver Rev. 2011; 63:1361-1370.

61. Valencia PM, et al. Effects of ligands with different water solubilities on self-assembly and properties of targeted nanoparticles. Biomaterials. 2011; 32:6226-6233. [PubMed: 21658757]

62. Timko BP, et al. Advances in drug delivery. Annu Rev Mater Res. 2011; 41:1-20.

63. Ruoslahti E. Peptides as targeting elements and tissue penetration devices for nanoparticles. Adv Mater. 2012; 24:3747-3756. [PubMed: 22550056] 
64. Sengupta S, et al. Temporal targeting of tumour cells and neovasculature with a nanoscale delivery system. Nature. 2005; 436:568-572. [PubMed: 16049491]

65. Ji T, et al. Using functional nanomaterials to target and regulate the tumor microenvironment: diagnostic and therapeutic applications. Adv Mater. 2013; 25:3508-3525. [PubMed: 23703805]

66. Waite C, Roth C. Nanoscale drug delivery systems for enhanced drug penetration into solid tumors: Current progress and opportunities. Crit Rev Biomed Eng. 2012; 40:21-41. [PubMed: 22428797]

67. Silva JM, et al. Immune system targeting by biodegradable nanoparticles for cancer vaccines. J Control Release. 2013; 168:179-199. [PubMed: 23524187]

68. Hou KK, et al. Mechanisms of Nanoparticle-Mediated siRNA Transfection by Melittin-Derived Peptides. ACS Nano. 2013; 7:8605-8615. [PubMed: 24053333]

69. Bocci G, et al. The pharmacological bases of the antiangiogenic activity of paclitaxel. Angiogenesis. 2013; 16:481-492. [PubMed: 23389639]

70. Goodman TT, et al. Increased nanoparticle penetration in collagenase-treated multicellular spheroids. Int J Nanomedicine. 2007; 2:265-274. [PubMed: 17722554]

71. Zhu L, Torchilin VP. Stimulus-responsive nanopreparations for tumor targeting. Integr Biol. 2013; 5:96-107.

72. Kost J, Langer R. Responsive polymeric delivery systems. Adv Drug Deliver Rev. 2012; 64:327341.

73. Kumari A, et al. Biodegradable polymeric nanoparticles based drug delivery systems. Colloid Surf B. 2010; 75:1-18.

74. Sailor MJ, Park JH. Hybrid nanoparticles for detection and treatment of cancer. Adv Mater. 2012; 24:3779-3802. [PubMed: 22610698]

75. Danhier F, et al. To exploit the tumor microenvironment: Passive and active tumor targeting of nanocarriers for anti-cancer drug delivery. J Control Release. 2010; 148:135-146. [PubMed: 20797419]

76. Lin K, et al. Nanoparticles that sense thrombin activity as synthetic urinary biomarkers of thrombosis. ACS Nano. 2013:9001-9009. [PubMed: 24015809]

77. Olson ES, et al. Activatable cell penetrating peptides linked to nanoparticles as dual probes for in vivo fluorescence and MR imaging of proteases. Proc Natl Acad Sci USA. 2010; 107:4311-4316. [PubMed: 20160077]

78. Cole AJ, et al. Cancer theranostics: the rise of targeted magnetic nanoparticles. Trends Biotechnol. 2011; 29:323-332. [PubMed: 21489647]

79. Derfus, aM, et al. Remotely triggered release from magnetic nanoparticles. Adv Mater. 2007; 19:3932-3936.

80. McCarthy JR, Weissleder R. Multifunctional magnetic nanoparticles for targeted imaging and therapy. Adv Drug Deliver Rev. 2008; 60:1241-1251.

81. Mo S, et al. Ultrasound-enhanced drug delivery for cancer. Expert Opin Drug Deliv. 2012; 9:15251538. [PubMed: 23121385]

82. Bagley AF, et al. Plasmonic photothermal heating of intraperitoneal tumors through the use of an implanted near-infrared source. ACS Nano. 2013; 7:8089-8097. [PubMed: 23961973]

83. von Maltzahn G, et al. Computationally guided photothermal tumor therapy using long-circulating gold nanorod antennas. Cancer Res. 2009; 69:3892-3900. [PubMed: 19366797]

84. Cheng Z, et al. Multifunctional nanoparticles: cost versus benefit of adding targeting and imaging capabilities. Science. 2012; 338:903-910. [PubMed: 23161990]

85. Eldar-Boock A, et al. Nano-sized polymers and liposomes designed to deliver combination therapy for cancer. Curr Opin Biotechnol. 2013; 24:682-689. [PubMed: 23726153]

86. Greco F, Vicent MJ. Combination therapy: opportunities and challenges for polymer-drug conjugates as anticancer nanomedicines. Adv Drug Deliver Rev. 2009; 61:1203-1213.

87. Nam J, et al. pH-responsive assembly of gold nanoparticles and spatiotemporally concerted drug release for synergistic cancer therapy. ACS Nano. 2013:3388-3402. [PubMed: 23530622]

88. von Maltzahn G, et al. Nanoparticle self-assembly gated by logical proteolytic triggers. J Am Chem Soc. 2007; 129:6064-6065. [PubMed: 17447766] 
89. Anglin EJ, et al. Porous silicon in drug delivery devices and materials. Adv Drug Deliver Rev. 2008; 60:1266-1277.

90. Serda RE, et al. Multi-stage delivery nano-particle systems for therapeutic applications. Biochim Biophys Acta. 2011; 1810:317-329. [PubMed: 20493927]

91. Park JH, et al. Cooperative nanoparticles for tumor detection and photothermally triggered drug delivery. Adv Mater. 2010; 22:880-885. [PubMed: 20217810]

92. Godin B, et al. An integrated approach for the rational design of nanovectors for biomedical imaging and therapy. Adv Genet. 2010; 69:31-64. [PubMed: 20807601]

93. Ren Y, et al. Identification and characterization of receptor-specific peptides for siRNA delivery. ACS Nano. 2012; 6:8620-8631. [PubMed: 22909216]

94. Cooper S, et al. Predicting protein structures with a multiplayer online game. Nature. 2010; 466:756-760. [PubMed: 20686574]

95. Martinez-Veracoechea FJ, Frenkel D. Designing super selectivity in multivalent nano-particle binding. Proc Natl Acad Sci U S A. 2011

96. Florence AT. Reductionism and complexity in nanoparticle-vectored drug targeting. J Control Release. 2012; 161:399-402. [PubMed: 22100439]

97. Anderson DG, et al. A combinatorial library of photocrosslinkable and degradable materials. Adv Mater. 2006; 18:2614-2618.

98. Green J, et al. A combinatorial polymer library approach yields insight into nonviral gene delivery. Acc Chem Res. 2008; 41

99. Valencia PM, et al. Microfluidic platform for combinatorial synthesis and optimization of targeted nanoparticles for cancer therapy. ACS Nano. 2013; 7:10671-10680. [PubMed: 24215426]

100. Douglas SM, et al. Self-assembly of DNA into nanoscale three-dimensional shapes. Nature. 2009; 459:414-418. [PubMed: 19458720]

101. Douglas SM, et al. A logic-gated nanorobot for targeted transport of molecular payloads. Science. 2012; 335:831-834. [PubMed: 22344439]

102. Iinuma R, et al. Polyhedra self-assembled from DNA tripods and characterized with 3D DNAPAINT. Science. 2014; 344:65-69. [PubMed: 24625926]

103. Maune HT, et al. Self-assembly of carbon nanotubes into two-dimensional geometries using DNA origami templates. Nat Nanotechnol. 2010; 5:61-66. [PubMed: 19898497]

104. Amornphimoltham $P$, et al. Intravital microscopy as a tool to study drug delivery in preclinical studies. Adv Drug Deliver Rev. 2011; 63:119-128.

105. Hak S, et al. Intravital microscopy in window chambers: a unique tool to study tumor angiogenesis and delivery of nanoparticles. Angiogenesis. 2010; 13:113-130. [PubMed: 20623252]

106. Albanese A, et al. Tumour-on-a-chip provides an optical window into nanoparticle tissue transport. Nat Commun. 2013; 4:2718. [PubMed: 24177351]

107. Lanza, R., et al. Principles of tissue engineering. Academic press; 2011.

108. Miller JS, et al. Rapid casting of patterned vascular networks for perfusable engineered threedimensional tissues. Nat Mater. 2012; 11:768-774. [PubMed: 22751181] 


\section{Highlights}

Nanoparticle designs and interactions in the body determine their individual behavior.

The collective behavior of nanoparticles can be exploited to improve performance

Bio-inspired mechanisms of cooperation include self-assembly and communication.

Systems approach builds on new nanomaterials, biological insight, and computation. 


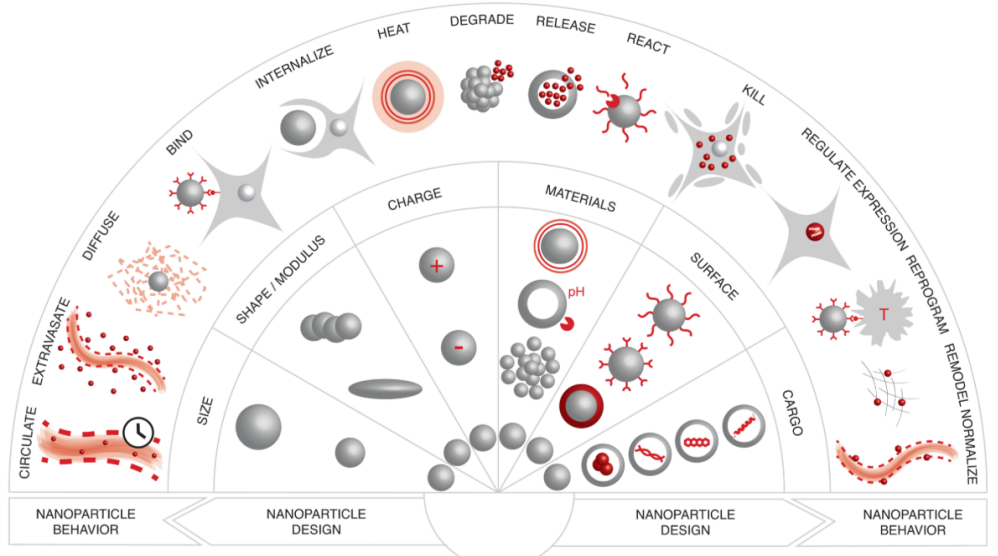

Figure 1.

Nanoparticle Behaviors. Nanoparticle designs, in terms of size, shape, modulus, charge, material, surface and cargo, as well as their interactions in the body, determine their individual behavior. 


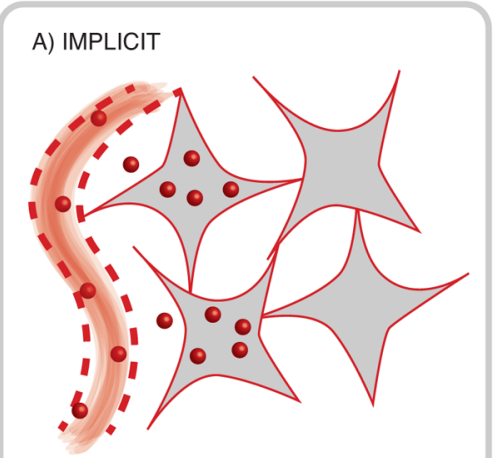

IMPROVE TISSUE DISTRIBUTION

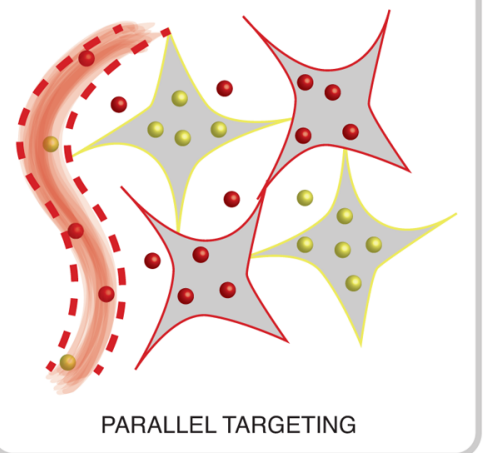

B) DIRECT

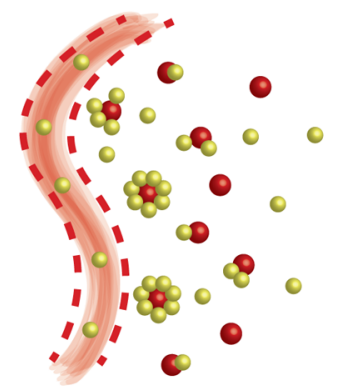

SELF-ASSEMBLE

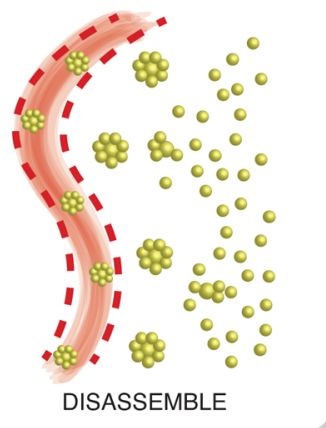

C) STIGMERGIC

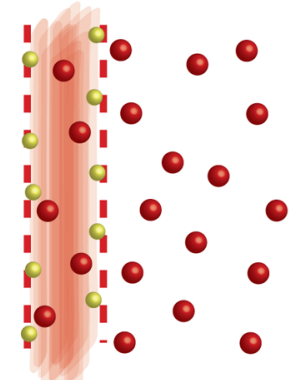

NORMALIZE / PERFUSE

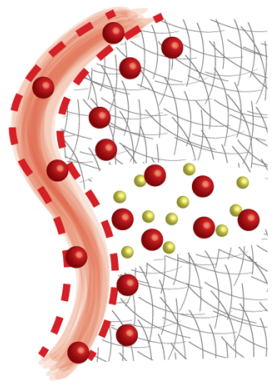

MODIFY TISSUE

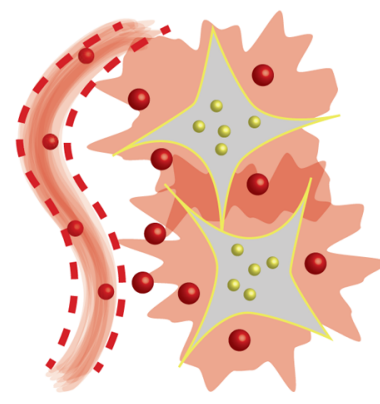

BIOLOGICAL SIGNALING

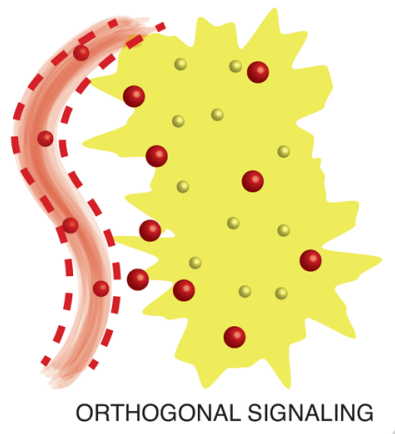

Figure 2.

Mechanisms of Cooperation in Nanosystems. A) Implicit cooperation: Each nanoparticle works individually to improve the overall tissue distribution (top) or work as a combination therapy (bottom). B) Direct cooperation: Physical interactions enable nanoparticles to selfassemble (top) and disassemble (bottom) towards improved nanoparticle transport and retention in tumors. C) Stigmergic cooperation: Nanoparticles interact through the environment by either modifying the environment to improve the transport of secondary nanoparticles (left), or by depositing a signal (right). Signaling nanoparticles can emit energy and chemicals, or coerce a biological response from the tumor environment, that can be received by a secondary nanoparticle. 


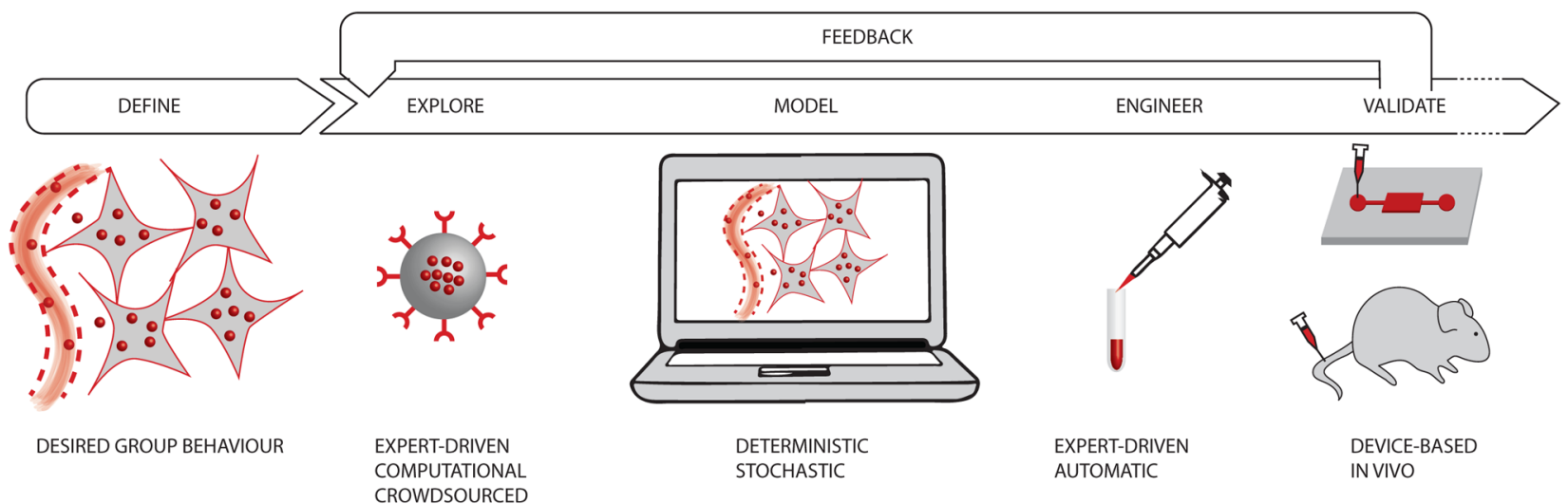

Figure 3 .

Systems Approach Towards Nanomedicine. A systems approach could help design the individual nanoparticles that would give rise to desired cooperative behaviors. This requires advances in computational exploration, modeling and the fast prototyping of experiments before validation in vivo. 